Functional Analysis and Its Applications, Vol. 43, No. 1, pp. 30-43, 2009

Translated from Funktsional'nyi Analiz i Ego Prilozheniya, Vol. 43, No. 1, pp. 37-54, 2009

Original Russian Text Copyright (c) by Bernhard Krötz

\title{
Horospherical Transform on Real Symmetric Varieties: Kernel and Cokernel
}

\author{
Bernhard Krötz
}

Received May 14, 2007

ABstRACT. In this paper, we define a horospherical transform for a semisimple symmetric space $Y$. A natural double fibration is used to assign a more geometrical space $\Xi$ of horospheres to $Y$. The horospherical transform relates certain integrable analytic functions on $Y$ to analytic functions on $\Xi$ by fiber integration. We determine the kernel of the horospherical transform and establish that the transform is injective on functions belonging to the most continuous spectrum of $Y$.

KEY WORDS: semisimple symmetric space, horospherical transform, Fourier transform, Plancherel theorem.

\section{Introduction}

This paper is about the horospherical (Radon) transform on semisimple groups and their homogeneous spaces. This transform was introduced by Gelfand and Graev in their seminal paper [6], where they treat the case of a complex semisimple group in detail and lay the foundation for considering the horospherical transform for other homogeneous spaces, in particular, Riemannian symmetric spaces of noncompact type.

The aim of this paper is to define the horospherical transform for an arbitrary semisimple symmetric space and establish its basic properties.

Let us turn to the subject proper of this paper. By $Y=G / H$ we denote a semisimple irreducible real symmetric variety (space).*

We consider the space $L^{2}(Y)$ of functions on $Y$ square integrable with respect to the $G$-invariant measure. This Hilbert space has the natural decomposition

$$
L^{2}(Y)=L_{\mathrm{mc}}^{2}(Y) \oplus L_{\mathrm{mc}}^{2}(Y)^{\perp}
$$

into the most continuous part and its orthogonal complement.

We also need the space $\mathscr{A}:=L^{1}(Y)^{\omega}$ of analytic vectors of the left regular representation of $G$ on $L^{1}(Y)$. Further, we set

$$
\mathscr{A}_{\mathrm{mc}}:=\mathscr{A} \cap L_{\mathrm{mc}}^{2}(Y) \text { and } \quad \mathscr{A}_{\mathrm{mc}}^{\perp}:=\mathscr{A} \cap L_{\mathrm{mc}}^{2}(Y)^{\perp} .
$$

We believe that $\mathscr{A}_{\mathrm{mc}}$ is dense in $L^{2}(Y)_{\mathrm{mc}}$ and $\mathscr{A}_{\mathrm{mc}}^{\perp}$ is dense in the subspace of $L^{2}(Y)_{\mathrm{mc}}^{\perp}$ corresponding to the principal series representations induced from integrable representations of the corresponding Levi subgroups. It would be of interest to prove this fact and similar facts for the case in which $\mathscr{A}$ is replaced by other function spaces.

We are interested in the open domain $\Xi=G /(M \cap H) N$ in the parameter space of generic real horospheres, where $M A N$ is a minimal $\sigma \theta$-invariant** parabolic subgroup of $G$.

By $C_{0}^{\omega}(\Xi)$ we denote the space of analytic functions on $\Xi$ vanishing at infinity. In this paper, we prove the following facts:

- The mapping

$$
\mathscr{R}: \mathscr{A} \rightarrow C_{0}^{\omega}(\Xi), \quad f \mapsto\left(g M_{H} N \mapsto \int_{N} f(g n H) d n\right),
$$

${ }^{*}$ This means that $G$ is a connected real semisimple Lie group and $H$ is the fixed point subgroup of an involutive automorphism $\sigma$ of $G$ such that there does not exist a $\sigma$-stable normal subgroup $L$ of $G$ with $0<\operatorname{dim} L<\operatorname{dim} G$.

${ }^{* *} \theta$ is a Cartan involution commuting with $\sigma$. 
is well defined. (We refer to $\mathscr{R}$ as the (minimal) horospherical transform.)

- $\left.\mathscr{R}\right|_{\mathscr{A} \mathrm{\perp c}}=0$.

- $\left.\mathscr{R}\right|_{\mathscr{A} \mathrm{mc}} \cap \mathscr{S}(Y)$ is injective.*

Acknowledgements. The above results are motivated by discussions with Simon Gindikin during my stay at IAS in October 2006. I am happy to acknowledge his input and the hospitality of IAS.

I would like to thank Henrik Schlichtkrull for pointing out a mistake and several inaccuracies in an earlier version of the paper. Also I thank the anonymous referee for pointing out a mistake and for his useful requests for more detail.

\section{Real Symmetric Varieties}

2.1. Notation. The objective of this section is to introduce notation and recall some facts regarding real symmetric varieties.

Let $G_{\mathbb{C}}$ be a simply connected complex linear algebraic group whose Lie algebra $\mathfrak{g}_{\mathbb{C}}$ is semisimple. We fix a real form $G$ of $G_{\mathbb{C}}$; this means that $G$ is the fixed point set of an involutive antiholomorphic automorphism $\sigma$ of $G_{\mathbb{C}}$ and that the complexification of $\mathfrak{g}$, the Lie algebra of $G$, coincides with $\mathfrak{g}_{\mathbb{C}}$.

Now let $\tau$ be a second involutive automorphism of $G_{\mathbb{C}}$ commuting with $\sigma$. In particular, $\tau(G)=$ $G$. We write $H_{\mathbb{C}}:=G_{\mathbb{C}}^{\tau}$ and $H:=G^{\tau}$ for the respective fixed point groups of $\tau$ in $G_{\mathbb{C}}$ and $G$. Note that $H_{\mathbb{C}}$ is always connected but $H$ is usually not; the simplest example of $\left(G_{\mathbb{C}}, G\right)=$ $(\mathrm{Sl}(2, \mathbb{C}), \mathrm{Sl}(2, \mathbb{R}))$ and $\left(H_{\mathbb{C}}, H\right)=(\mathrm{SO}(1,1 ; \mathbb{C}), \mathrm{SO}(1,1 ; \mathbb{R}))$ illustrates the situation.

Using $G$ and $H$, we form the homogeneous space $Y=G / H$; we refer to $Y$ as a real (semisimple) symmetric variety (or space). From now on, we denote the standard base point in $Y$ by $y_{o}=H$. We write $Y_{\mathbb{C}}=G_{\mathbb{C}} / H_{\mathbb{C}}$ for the complexification of $Y$ and, whenever convenient, view $Y$ as a subspace of $Y_{\mathbb{C}}$ via the embedding

$$
Y \hookrightarrow Y_{\mathbb{C}}, \quad g H \mapsto g H_{\mathbb{C}}
$$

At this point, it is useful to introduce notation for infinitesimal objects. Lie groups will always be denoted by uppercase Latin letters, e.g., $G, H, K$, etc., and the corresponding Lie algebras by lowercase German letters, e.g., $\mathfrak{g}, \mathfrak{h}, \mathfrak{k}$, etc. If $\tau$ is an automorphism of a Lie group $G$, then we use the same symbol $\tau$ for the derived automorphism $d \tau(\mathbf{1})$ of the Lie algebra $\mathfrak{g}$. By $\mathfrak{q}$ we denote the $(-1)$-eigenspace of $\tau$ in $\mathfrak{g}$. Note that the $H$-module $\mathfrak{q}$ can naturally be identified with the tangent space $T_{y_{o}} Y$ of $Y$ at the base point.

From now on, we assume that $Y$ is irreducible, i.e., that the only $\tau$-invariant ideals in $\mathfrak{g}$ are $\{0\}$ and $\mathfrak{g}$. In practice, this means that $G$ is simple except for the case $G / H=(H \times H) / H \simeq H$.

Recall that the maximal compact subgroups $K \subset G$ are in a one-to-one correspondence with the Cartan involutions $\theta: G \rightarrow G$. The correspondence is given by $K=G^{\theta}$. We form the Riemannian symmetric space $X=G / K$ of noncompact type and denote by $x_{o}=K$ the standard base point of $X$. As before, we write $\theta$ for the derived involution on $\mathfrak{g}$. Let $\mathfrak{p} \subset \mathfrak{g}$ be the $(-1)$-eigenspace of $\theta$. We identify the $K$-module $\mathfrak{p}$ with $T_{x_{o}} X$.

According to Berger, we can (and will) assume that $K$ is $\tau$-invariant. This implies that both $\mathfrak{p}$ and $\mathfrak{k}$ are $\tau$-invariant. Let us fix a maximal Abelian subspace $\mathfrak{a} \subset \mathfrak{q} \cap \mathfrak{p}$. Note that $\mathfrak{a}$ is unique modulo conjugation by $H \cap K$; see [13, Lemma 7.1.5]. Set $A=\exp (\mathfrak{a})$.

Next, consider the centralizer $Z_{G}(A)$ of $A$. First, we note that $Z_{G}(A)$ is reductive and admits the natural decomposition

$$
Z_{G}(A)=A \times M
$$

(cf. [10, Proposition 7.82 (a)]). The Lie algebra of $M$ is $\mathfrak{m}=\mathfrak{z} \mathfrak{g}(\mathfrak{a}) \cap \mathfrak{a}^{\perp}$, where $\mathfrak{a}^{\perp}$ is the orthogonal complement of $\mathfrak{a}$ in $\mathfrak{g}$ with respect to the Cartan-Killing form $\kappa$ on $\mathfrak{g}$. If $M_{0}$ is the connected

\footnotetext{
* $\mathscr{S}(Y)$ is the Schwartz space of rapidly decaying functions.
} 
component of unity in $M$, then $M=M_{0} F$, where $F \subset M \cap K$ is an elementary finite 2-group (cf. [10, Proposition 7.82 (d) and Theorem 7.52]).

Remark 2.1. If $\mathfrak{a}$ is maximal Abelian subspace in $\mathfrak{p}$, then, as follows from the explicit description of $F$ in [10, Theorem 7.52], $F \subset H$. In general, however, the $\tau$-invariant group $F$ is not contained in $H$.

We write $\mathfrak{m}_{\mathrm{ns}}$ for the noncompact semisimple part of $\mathfrak{m}$ and note that

$$
\mathfrak{m}_{\mathrm{ns}} \subset \mathfrak{h}
$$

(cf. [13, Lemma 7.1.4]). Set $M_{H}=M \cap H=Z_{H}(A)$, and let $\mathfrak{m}=\mathfrak{m}_{h}+\mathfrak{m}_{q}$ be the decomposition of $\mathfrak{m}$ into the $( \pm 1)$-eigenspaces. Note that $\mathfrak{m}_{h}$ is the Lie algebra of $M_{H}$. Then (2.1) implies that $\mathfrak{m}_{q} \subset \mathfrak{k}$ and hence the set $M_{q}=\exp \left(\mathfrak{m}_{q}\right)$ is compact. Moreover,

$$
M / F=M_{H} M_{q} / F, \text { and } M_{H} \cap M_{q} \text { is discrete. }
$$

Consider the root space decomposition of $\mathfrak{g}$ with respect to $\mathfrak{a}$. For $\alpha \in \mathfrak{a}^{*}$, let

$$
\mathfrak{g}^{\alpha}=\{X \in \mathfrak{g} \mid \forall Y \in \mathfrak{a}[Y, X]=\alpha(Y) X\}
$$

and

$$
\Sigma=\left\{\alpha \in \mathfrak{a}^{*} \backslash\{0\} \mid \mathfrak{g}^{\alpha} \neq\{0\}\right\} .
$$

It is known that $\Sigma$ is a (possibly nonreduced) root system, cf. [13, Proposition 7.2.1]. Hence we can fix a positive root system $\Sigma^{+} \subset \Sigma$ and define the corresponding nilpotent subalgebra

$$
\mathfrak{n}:=\bigoplus_{\alpha \in \Sigma^{+}} \mathfrak{g}^{\alpha} .
$$

Set $N:=\exp (\mathfrak{n})$. Note that $\tau(\mathfrak{n})=\theta(\mathfrak{n})$. We have the decomposition

$$
\mathfrak{g}=\mathfrak{a} \oplus \mathfrak{m} \oplus \mathfrak{n} \oplus \tau(\mathfrak{n}) .
$$

We shift our focus to the real flag manifold of $G$ associated with $A$ and $\Sigma^{+}$. We define $P_{\min }:=$ $M A N$ and note that $P_{\min }$ is a minimal $\theta \tau$-invariant parabolic subgroup of $G$.

The decomposition of the flag manifold $G / P_{\min }$ into open $H$-orbits is important in the theory of $H$-spherical representations of $G$. To describe this decomposition, we need some facts about Weyl groups.

Let $\mathscr{W}$ be the Weyl group of the root system $\Sigma$. The Weyl group admits the analytic realization

$$
\mathscr{W}=N_{K}(\mathfrak{a}) / Z_{K}(\mathfrak{a})
$$

and contains the natural subgroup

$$
\mathscr{W}_{H}:=N_{H \cap K}(\mathfrak{a}) / Z_{H \cap K}(\mathfrak{a}) .
$$

Knowing $\mathscr{W}$ and $\mathscr{W}_{H}$, we can find the decomposition of $G$ into open $\left(P_{\min } \times H\right)$-cosets (cf. [12]),

$$
G \doteq \amalg_{w \in \mathscr{W} / \mathscr{W}_{H}} P_{\min } w H,
$$

where $\doteq$ stands for equality up to a finite union of strictly lower-dimensional $\left(P_{\min } \times H\right)$-orbits.

2.2. Horospheres. This subsection deals with horospheres on the symmetric variety $Y$. By a (generic) horosphere on $Y$ we understand an orbit of maximum dimension (i.e. $\operatorname{dim} N$ ) of a subgroup conjugate to $N$. The set of all horospheres will be denoted by $\operatorname{Hor}(Y)$. Note that $G$ naturally acts on $\operatorname{Hor}(Y)$ on the left.

Our goal is to show that $\operatorname{Hor}(Y)$ is a connected analytic manifold. To this end, we define

$$
G_{h}:=\left\{x \in G \mid N x \cdot y_{o} \in \operatorname{Hor}(Y)\right\}
$$

and note the following immediate things:

- $G_{h}$ is open, right $H$-invariant, and left $P_{\min }$-invariant.

- $G_{h}$ contains the open $P_{\min } \times H$-cosets $P_{\min } w H$, where $w \in \mathscr{W} / \mathscr{W}_{H}$; see (2.2). In particular, $G_{h}$ is dense.

- $\operatorname{Hor}(Y)=\left\{g N x \cdot y_{o} \mid g \in G, x \in G_{h}\right\}$. 
- (Infinitesimal characterization) $G_{h}=\left\{x \in G \mid \mathscr{A} d\left(x^{-1}\right) \mathfrak{n} \cap \mathfrak{h}=\{0\}\right\}$.

Remark 2.2. The set $G_{h}$ is in general larger then the disjoint union $\bigcup_{w \in \mathscr{W} / \mathscr{W}_{H}} P_{\min } w H$ (which is an open dense subset of $G$ ). In particular, it is connected, as we show below. For example, if $G=\mathrm{Sl}(2, \mathbb{R}), H=\mathrm{SO}(1,1 ; \mathbb{R})$, and $P_{\min }$ is the subgroup of upper triangular matrices with determinant 1 , then $\mathfrak{h}$ and $\mathfrak{n}$, which are both one-dimensional, cannot be conjugate. Thus, by the infinitesimal characterization given above, one has $G_{h}=G$ in this case.

Next, we provide charts for $\operatorname{Hor}(Y)$. To this end, we introduce the $G$-manifold $\Xi=G / M_{H} N$ and define the $G$-equivariant mapping

$$
E: \Xi \rightarrow \operatorname{Hor}(Y), \quad \xi=g M_{H} N \mapsto E(\xi)=g N \cdot y_{o} .
$$

As in [9, Proposition 2.1], one verifies that $E$ is an injection. Now we move our chart by elements $x \in G_{h}$. Set $L:=M A, L^{x}=x^{-1} L x, L_{H}^{x}:=L^{x} \cap H, N^{x}:=x^{-1} N x$, and $\Xi_{x}:=G / L_{H}^{x} N^{x}$. Then the mapping

$$
E_{x}: G / L_{H}^{x} N^{x} \rightarrow \operatorname{Hor}(Y), \quad g L^{x} N^{x} \mapsto g N^{x} \cdot y_{o}
$$

is $G$-equivariant and injective. It is immediate that $\left(E_{x}, \Xi_{x}\right)_{x \in G_{h}}$ form an analytic atlas for $\operatorname{Hor}(Y)$.

Lemma 2.3. $\operatorname{Hor}(Y)$ is connected.

Proof. It suffices to show that $G_{h}$ is connected. We know that $G_{h}$ is an open dense $\left(P_{\min } \times H\right)$ invariant subset of $G$. Now there are only finitely many orbits of $P_{\min } \times H$ in $G$, and these are described explicitly; see [12].

Since $G_{h}$ contains all open orbits, it suffices to show that $G_{h}$ contains all codimension one orbits. This, in turn, follows from the explicit description of all orbits in [12, Th. 3 (i)]: if $P_{\min } c H \subset G$ is not open, then [12] implies that $\mathscr{A} d(c) \mathfrak{a} \cap \mathfrak{h} \neq\{0\}$. In particular, if $P_{\min } c H$ is of codimension one, then $\mathscr{A} d(c)(\mathfrak{m}+\mathfrak{a}+\mathfrak{n}) \cap \mathfrak{h}=\mathscr{A} d(c) \mathfrak{a} \cap \mathfrak{h}$, and therefore, $\mathscr{A} d(c) \mathfrak{n} \cap \mathfrak{h}=\{0\}$. Our infinitesimal characterization completes the proof.

Finally, we discuss polar coordinates on $\Xi$. The mapping

$$
K /\left(M_{H} \cap K\right) \times A \rightarrow \Xi, \quad\left(k\left(M_{H} \cap K\right), a\right) \mapsto k a M_{H} N
$$

is a diffeomorphism. We shall often treat $A$ as subspace of $\Xi$ with the embedding

$$
A \hookrightarrow \Xi, \quad a \mapsto a M_{H} N .
$$

\section{Function Spaces and Definition of the Horospherical Transform}

Consider the left regular representation $L$ of $G$ on $L^{1}(Y)$ :

$$
[L(g) f](y)=f\left(g^{-1} y\right) \quad(y \in Y)
$$

for $g \in G$ and $f \in L^{1}(Y)$. Let $\mathscr{A}:=L^{1}(Y)^{\omega}$ be the subspace of analytic vectors for $L$. The condition $f \in \mathscr{A}$ means that $f \in C^{\omega}(Y)$ and there exists an open neighborhood $U$ of $\mathbf{1}$ in $G_{\mathbb{C}}$ such that $f$ extends holomorphically to the subset

$$
U G \cdot y_{o} \subset Y_{\mathbb{C}}=G_{\mathbb{C}} / H_{\mathbb{C}}
$$

(here we assume that $Y$ is embedded in $Y_{\mathbb{C}}$ via $g H \mapsto g H_{\mathbb{C}}$ ) in such a way that

$$
\sup _{c \in C}\|f(c \cdot)\|_{L^{1}(Y)}<\infty
$$

for all compact $C \subset U$ (cf. [7, Proposition A.2.1]). For an open neighborhood $U$ of $\mathbf{1}$ in $G_{\mathbb{C}}$, we denote by $\mathscr{A}_{U}$ the space of holomorphic functions on $U G \cdot y_{o}$ that satisfy (3.1) for all compact $C \subset U$. Note that $\mathscr{A}_{U}$ can be viewed as a closed subspace of $\mathscr{O}\left(U, L^{1}(Y)\right)$ and hence is a Fréchet space. Moreover,

$$
\mathscr{A}=\bigcup_{U} \mathscr{A}_{U}
$$


with the continuous embeddings

$$
\mathscr{A}_{U} \rightarrow \mathscr{A}_{V},\left.\quad f \mapsto f\right|_{V G \cdot y_{o}}
$$

for $V \subset U$. This way, we equip $\mathscr{A}$ with the structure of a locally convex space.

Note that the representation $L$ defines an analytic action of $G$ on $\mathscr{A}$.

3.1. Definition of the horospherical transform. We start from a crucial technical fact.

Lemma 3.1. Let $f \in \mathscr{A}$. Then the following assertions hold:

(i) $\sup _{\substack{a \in A \\ k \in K}} \int_{N} \mid f\left(\right.$ kan $\left.\cdot y_{o}\right) \mid d n<\infty$.

(ii) $\sup _{\substack{a \in A \\ k \in K}} a^{-2 \rho} \int_{N}\left|f\left(k n a \cdot y_{o}\right)\right| d n<\infty$.

Proof. (i) Let $f \in \mathscr{A}$. Let $B_{\mathfrak{a}} \subset \mathfrak{a}$ and $B_{\mathfrak{n}} \subset \mathfrak{n}$ be balls centered at zero. Set

$$
U_{A}:=\exp \left(B_{\mathfrak{a}}+i B_{\mathfrak{a}}\right) \subset A_{\mathbb{C}}, \quad U_{N}:=\exp \left(i B_{\mathfrak{n}}\right) \exp \left(B_{\mathfrak{n}}\right) \subset N_{\mathbb{C}} .
$$

If $B_{\mathfrak{a}}$ and $B_{\mathfrak{n}}$ are sufficiently small, then $f$ extends to a holomorphic function in a neighborhood of $K U_{N} U_{A} G \cdot y_{o}$ such that

$$
\sup _{c \in K U_{N} U_{A}}\|f(c \cdot)\|_{L^{1}(Y)}<\infty .
$$

To simplify the notation, let us assume that $M \subset H$; this does not reduce generality, since the complement $M_{q} F$ of $M_{H}$ in $M$ is compact by (2.1). Then the mapping

$$
N A \rightarrow Y, \quad n a \mapsto n a \cdot y_{o},
$$

is an open embedding. In particular, $N A \cdot y_{o} \subset Y$ is open. It follows that $U_{A} U_{N} A N \cdot y_{o} \subset Y_{\mathbb{C}}$ is open, and we can assume that $U_{A} U_{N} A N \subset A_{\mathbb{C}} N_{\mathbb{C}}$ is embedded in $Y_{\mathbb{C}}$. Take a $k \in K$ and define a holomorphic function $F$ on $U_{N} U_{A} A N$ by setting $F(z):=f\left(k z \cdot y_{o}\right)$. In particular, we obtain a constant $C>0$ such that

$$
|F(a n)| \leqslant C \int_{U_{N} U_{A}}\left|F\left(n^{\prime} a^{\prime} a n\right)\right| d a^{\prime} d n^{\prime}
$$

for all an $\in A N$, where $d a^{\prime}$ and $d n^{\prime}$ are Haar measures on $A_{\mathbb{C}}$ and $N_{\mathbb{C}}$ (Bergman's estimate). Let $d y$ be the Haar measure on $Y$. Note that the restriction of $d y$ to $A N$ is equal to $d a d n$, where $d a$ and $d n$ are the Haar measures on $A$ and $N$, respectively. Therefore,

$$
\begin{aligned}
\int_{N}|F(a n)| d n & \leqslant C \int_{U_{N} U_{A}} \int_{N}\left|F\left(n^{\prime} a^{\prime} a n\right)\right| d a^{\prime} d n^{\prime} d n \\
& \leqslant C \int_{U_{N}} \int_{B_{\mathfrak{a}}} \int_{A} \int_{N}\left|F\left(n^{\prime} \exp (i X) a^{\prime} a n\right)\right| d n^{\prime} d X d a^{\prime} d n \\
& \leqslant C \int_{U_{N}} \int_{B_{\mathfrak{a}}} \int_{Y}\left|f\left(k n^{\prime} \exp (i X) y\right)\right| d n^{\prime} d X d y \\
& \leqslant C \cdot \operatorname{vol}\left(U_{N}\right) \cdot \operatorname{vol}\left(B_{\mathfrak{a}}\right) \sup _{c \in K U_{A} U_{N}}\|f(c \cdot)\|_{L^{1}(Y)}<\infty
\end{aligned}
$$

Note that the last expression does not depend on $a \in A$ and $k \in K$. This proves (i). Now (ii) is just a change of variables away from (i):

$$
\int_{N}\left|f\left(k n a \cdot y_{o}\right)\right| d n=\int_{N}\left|f\left(k a a^{-1} n a \cdot y_{o}\right)\right| d n=a^{2 \rho} \int_{N}\left|f\left(k a n \cdot y_{o}\right)\right| d n .
$$

For any $f \in \mathscr{A}$, we define the horospherical transform $\mathscr{R}(f)$ of $f$ on $\Xi$ by setting

$$
\mathscr{R}(f)\left(g M_{H} N\right):=\int_{N} f\left(g n \cdot y_{o}\right) d n .
$$

By the previous lemma, the integral is absolutely convergent. 
Let $C_{0}^{\omega}(\Xi)$ be the space of analytic functions on $\Xi$ vanishing at infinity. In view of $(2.3)$, the condition that $F$ vanishes at infinity means that

$$
\lim _{\substack{a \rightarrow \infty \\ a \in A}} \sup _{\substack{k \in K \\ \text { a }}}\left|F\left(k a M_{H} N\right)\right|=0 .
$$

Proposition 3.2. The following assertions hold:

(i) For all $f \in \mathscr{A}$, one has $\mathscr{R}(f) \in C_{0}^{\omega}(\Xi)$.

(ii) The mapping $\mathscr{R}: \mathscr{A} \rightarrow L^{1}(\Xi)^{\omega} \subset C_{0}^{\omega}(\Xi)$ is continuous.

Proof. (i) As before, we can assume without loss of generality that $M \subset H$, because the part of $M$ not in $H$ is compact by (2.1). First, let us show that $F:=\left.\mathscr{R}(f)\right|_{A} \in C_{0}^{\omega}$. Indeed, since $f \in L^{1}(Y)$ and $\left.d y\right|_{A N}=d a d n$, it follows that $F \in L^{1}(A)$. Moreover, $F \in L^{1}(A)^{\omega}$; i.e., $F$ is an analytic vector for the regular representation of $A$ on $L^{1}(A)$. Therefore, the standard Sobolev lemma implies that $F \in C_{0}^{\omega}(A)$.

Finally, the introduction of the additional parameter $k$ varying in the compact group $K$ causes no further difficulties.

(ii) This follows from (i) and the last (and crucial) estimate in the proof of Lemma 3.1(i).

Remark 3.3. In fact, one can define the horospherical transform in such a way that the transform of any function will be defined on the entire horosphere space Hor $(Y)$. Let us return to the set $G_{h} \subset G$ and the parameter space $\Xi_{x}=G / L_{H}^{x} N^{x}$ for each $x \in G_{h}$. For every $f \in \mathscr{A}$, one then defines

$$
\mathscr{R}_{x}(f)\left(g L_{H}^{x} N^{x}\right)=\int_{N} f\left(g x^{-1} n x \cdot y_{o}\right) d n .
$$

As we have seen above, the resulting function $\mathscr{R}_{x}(f)$ lies in $C_{0}^{\omega}\left(\Xi_{x}\right) \cap L^{1}\left(\Xi_{x}\right)$. As a result, we obtain a well-defined $G$-mapping

$$
\mathscr{R}: \mathscr{A} \rightarrow C^{\omega}(\operatorname{Hor}(Y)) \text {. }
$$

\section{The Kernel of the Horospherical Transform: Discrete Spectrum}

In this section, we show that the intersection of the discrete spectrum of the representation of $G$ on $L^{2}(Y)$ with $\mathscr{A}$ lies in the kernel of $\mathscr{R}$. In fact, we show even more: the intersection of $\mathscr{A}$ with the orthogonal complement of the most continuous spectrum lies in the kernel.

Recall the minimal $\theta \tau$-invariant parabolic subgroup

$$
P_{\min }=M A N \text {. }
$$

In the sequel, we use the symbol $Q$ to denote a $\theta \tau$-invariant parabolic subgroup that contains $P_{\min }$. There are finitely many such subgroups. Let

$$
Q=M_{Q} A_{Q} N_{Q}
$$

be the standard factorization of $Q$. Note that

- $M_{Q} \supset M$

- $A_{Q} \subset A$

- $N_{Q} \subset N$

One says that two parabolic subgroups $Q$ and $Q^{\prime}$ are associated if there exists an $n \in N_{K}(A)$ such that $n A_{Q} n^{-1}=A_{Q^{\prime}}$. This induces an equivalence relation $\sim$ on parabolic subgroups, and we write $[Q]$ for the corresponding equivalence classes. If the context is clear, we simply omit the brackets and write $Q$ instead of $[Q]$.

It follows from the Plancherel theorem ([3], [5]) that

$$
L^{2}(Y)=\bigoplus_{Q \supset P_{\min } / \sim} L^{2}(Y)_{Q}
$$

where $L^{2}(Y)_{Q}=L^{2}(Y)_{[Q]}$ stands for the part corresponding to representations induced from $Q$ by discrete series representations of the groups $M_{Q} / M_{Q} \cap w H w^{-1}$, where $w$ runs over representatives of elements of $\mathscr{W} / \mathscr{W}_{H}$. 
Since $\mathscr{A} \subset C_{0}^{\omega}(Y)$ (see [11]), we observe that $\mathscr{A} \subset L^{2}(Y)$. However, $\mathscr{A}$ may not be dense in $L^{2}(Y)$ : it has no components in the part of $L^{2}(Y)_{Q}$ corresponding to the representations induced from nonintegrable discrete series representations of $M_{Q} / M_{Q} \cap w H w^{-1}$.

Let $\mathscr{A}_{Q}=L^{2}(Y)_{Q} \cap \mathscr{A}$. For the extreme choices of $Q$, we use the special notation

$$
L^{2}(Y)_{\mathrm{disc}}:=L^{2}(Y)_{G} \text { and } \quad L^{2}(Y)_{\mathrm{mc}}:=L^{2}(Y)_{P_{\min }}
$$

and speak of the discrete and most continuous parts, respectively, of the square integrable spectrum. In a similar way, we define $\mathscr{A}_{\text {disc }}$ and $\mathscr{A}_{\text {mc }}$. We believe that

$$
\mathscr{A}_{\mathrm{mc}} \subset L^{2}(Y)_{\mathrm{mc}} \text { is dense. }
$$

(The heuristic reason in favor of this is that $M / M \cap H$ is compact.)

Theorem 4.1. $\mathscr{R}\left(\mathscr{A}_{\text {disc }}\right)=\{0\}$.

Proof. The proof is the same as for the group space; see [14, Theorem 7.2.2] for a useful exposition.

Let $f \in \mathscr{A}_{\text {disc }}$. We have to show that $\mathscr{R}(f)=0$. Since $\mathscr{R}$ is continuous (Proposition 3.2), we can use standard density arguments to reduce the assertion to the case in which $f$ belongs to a single discrete series representation and is $K$-finite. Let

$$
V=\mathscr{U}\left(\mathfrak{g}_{\mathbb{C}}\right) f
$$

be the corresponding Harish-Chandra module, and set $T:=\left.\left[\left.\mathscr{R}\right|_{V}\right]\right|_{A}$. Then $T$ factors through the Jacquet module $j(V)=V / \mathfrak{n} V$. Recall that $j(V)$ is an admissible finitely generated $(M, \mathfrak{a})$-module. Hence

$$
\operatorname{dim} \mathscr{U}(\mathfrak{a}) T(f)<\infty
$$

Consequently,

$$
T(f)(a)=\sum_{\mu} a^{\mu} p_{\mu}(\log a) \quad(a \in A),
$$

where $\mu$ runs over a finite subset in $\mathfrak{a}_{\mathbb{C}}^{*}$ and $p_{\mu}$ is a polynomial (see [14, 8.A.2.10]). Since $T(f) \in$ $C_{0}^{\omega}(A)$, we conclude that $T(f)=0$, and hence $\mathscr{R}(f)=0$ by the $K$-finiteness of $f$.

As a consequence of the preceding theorem, we obtain the main result of this subsection.

Theorem 4.2. Let $Q \supsetneq P_{\min }$. Then $\mathscr{R}\left(\mathscr{A}_{Q}\right)=\{0\}$.

Proof. If $Q=G$, then this is just the previous theorem. The general case will be reduced to that. Suppose that $P_{\min } \subsetneq Q \subsetneq G$. We define $\Xi_{Q}=G /\left(M_{Q} \cap H\right) N_{Q}$ and, just as in (2.3), obtain a diffeomorphic parameterization $\left[K \times_{M_{Q} \cap K} M_{Q} / M_{Q} \cap H\right] \times A \rightarrow \Xi_{Q}$.

As in Section 3.1, we conclude that the mapping

$$
\mathscr{R}_{Q}: \mathscr{A} \rightarrow L^{1}\left(\Xi_{Q}\right)^{\omega}, \quad f \mapsto\left(g\left(M_{Q} \cap H\right) N_{Q} \mapsto \int_{N_{Q}} f(g n H) d n\right),
$$

is well-defined, $G$-equivariant, and continuous.

Next, observe that

$$
N=N_{Q} \rtimes N^{Q},
$$

where $\{\mathbf{1}\} \neq N^{Q} \subset M_{Q}$. As before, the mapping

$$
\mathscr{R}^{Q}: L^{1}\left(\Xi_{Q}\right)^{\omega} \rightarrow L^{1}(\Xi)^{\omega}, \quad f \mapsto\left(g M_{H} N \mapsto \int_{N^{Q}} f\left(g n\left(M_{Q} \cap H\right) N_{Q}\right) d n\right),
$$

is well-defined, equivariant, and continuous.

Now note that

$$
\mathscr{R}=\mathscr{R}^{Q} \circ \mathscr{R}_{Q} .
$$

Now let $f \in \mathscr{A}_{Q}$. Without loss of generality, we can assume that $f$ belongs to a wave packet induced from a discrete series representation $\sigma$ realized in $L^{2}\left(M_{Q} / M_{Q} \cap H\right)$. 
Note that $M_{Q} / M_{Q} \cap H$ is naturally embedded in $\Xi_{Q}$ and that the restrictions of functions in $L^{1}\left(\Xi_{Q}\right)^{\omega}$ to $M_{Q} / M_{Q} \cap H$ remain integrable. Hence the restriction of $F:=\mathscr{R}_{Q}(f)$ to $M_{Q} / M_{Q} \cap H$ is integrable as well.

We claim that $\left.F\right|_{M_{Q} / M_{Q} \cap H}$ belongs to the $\sigma$-isotypical class. First, note that $L^{1}\left(\Xi_{Q}\right)^{\omega} \subset$ $L^{2}\left(\Xi_{Q}\right)$. By stage induction,

$$
L^{2}\left(\Xi_{Q}\right)=\operatorname{Ind}_{\left(M_{Q} \cap H\right) N_{Q}}^{G} \operatorname{triv} \simeq \operatorname{Ind}_{M_{Q} N_{Q}}^{G} L^{2}\left(M_{Q} / M_{Q} \cap H\right) .
$$

Thus, if $L^{2}\left(M_{Q} / M_{Q} \cap H\right)=\int \frac{\oplus}{\widehat{M}_{Q}} m_{\pi} \mathscr{H}_{\pi} d \mu(\pi)$ is the Plancherel decomposition, then we have the $G$-module isomorphism

$$
L^{2}\left(\Xi_{Q}\right) \simeq \int_{\hat{M}_{Q}}^{\oplus} m_{\pi} \operatorname{Ind}_{M_{Q} N_{Q}}^{G} \mathscr{H}_{\pi} d \mu(\pi) .
$$

Now note that $A_{Q}$ acts on $\Xi_{Q}$ on the right and this action commutes with that of $G$. (See the next section for a detailed discussion for $Q=P_{\min }$.) This gives a further disintegration of the left regular representation $L_{Q}$ of $G$ on $L^{2}\left(\Xi_{Q}\right)$ :

$$
L_{Q} \simeq \int_{\widehat{M}_{Q}}^{\oplus} m_{\pi} \int_{i \mathfrak{a}_{Q}^{*}}^{\oplus} \operatorname{Ind}_{M_{Q} A_{Q} N_{Q}}^{G}\left[\pi \otimes\left(-\lambda-\rho_{Q}\right) \otimes \mathbf{1}\right] d \lambda d \mu(\pi) .
$$

Since $R_{Q}$ is $G$-equivariant, we conclude that $R_{Q}(f) \in \operatorname{Ind}_{M_{Q} N_{Q}}^{G}$.

Our claim, combined with the previous theorem, implies that

$$
\left.\mathscr{R}^{Q}(F)\right|_{M_{Q} / M_{H} N^{Q}}=0 .
$$

By the equivariance of $\mathscr{R}_{Q}$ and $\mathscr{R}^{Q}$, we can replace $f$ (and hence $F$ ) by any of its $G$-translates. Consequently, $\mathscr{R}^{Q}(F)=0$, as desired.

\section{Restriction of the Horospherical Transform to the Most Continuous Spectrum}

The objective of this section is to show that $\mathscr{R}$ is faithful on the most continuous spectrum.

Recall a few facts on the spectrum of the representation of $G$ on $L^{2}(\Xi)$ and the most continuous spectrum on $Y$. We start from the "horocyclic picture." The homogeneous space $\Xi$ carries a $G$-invariant measure. Consequently, left shifts by $G$ in the argument of a function on $\Xi$ define a unitary representation, say, $L$, of $G$ on $L^{2}(\Xi)$; namely,

$$
(L(g) f)(\xi)=f\left(g^{-1} \cdot \xi\right) \quad\left(f \in L^{2}(\Xi), g \in G, \xi \in \Xi\right) .
$$

It is important to note that the $G$-action on $\Xi$ admits a commuting right action of $A$,

$$
\xi \cdot a=g a M N \quad\left(\xi=g M_{H} N \in \Xi, a \in A\right),
$$

because $A$ normalizes $M_{H} N$. Therefore, the formula

$$
(R(a) f)(\xi)=a^{\rho} \cdot f(\xi \cdot a) \quad\left(f \in L^{2}(\Xi), a \in A, \xi \in \Xi\right)
$$

defines a unitary representation $\left(R, L^{2}(\Xi)\right)$ of $A$ commuting with $L$. Accordingly, we define the $A$-Fourier transform of an appropriate function $f$ on $\Xi$ by the formula

$$
\mathscr{F}_{A}(f)\left(\lambda, g M_{H} N\right):=\int_{A}[R(a) f]\left(g M_{H} N\right) a^{\lambda} d a \quad\left(\lambda \in i \mathfrak{a}^{*}\right) .
$$

For $\lambda \in \mathfrak{a}_{\mathbb{C}}^{*}$, we set

$$
\begin{aligned}
& L^{2}(\Xi)_{\lambda}:=\left\{f: G \rightarrow \mathbb{C} \mid f \text { is measurable, } f(\cdot \operatorname{man})=a^{-\rho-\lambda} f(\cdot) \forall \operatorname{man} \in M_{H} A N,\right. \\
& \left.\qquad \int_{K}|f(k)|^{2} d k<\infty\right\} .
\end{aligned}
$$


Likewise, by $C^{\infty}(\Xi)_{\lambda}$ we denote the space of smooth elements in $L^{2}(\Xi)_{\lambda}$. The disintegration of $L^{2}(\Xi)$ is then given by

$$
L^{2}(\Xi) \simeq \int_{i \mathfrak{a}^{*}}^{\oplus} L^{2}(\Xi)_{\lambda} d \lambda
$$

where the isomorphism is given by the $A$-Fourier transform

$$
f \mapsto\left(\lambda \mapsto \mathscr{F}_{A}(f)(\lambda, \cdot)\right)
$$

Now recall the Plancherel decomposition for the most continuous spectrum (cf. [1]). First, we make some general remarks. For a representation $\pi$ of a group $L$ on some topological vector space $V$, by $\pi^{*}$ we denote the dual representation on the (strong) topological dual $V^{*}$ of $V$.

Let $\sigma \in \widehat{M / M_{H}}$, and let $V_{\sigma}$ be the unitary representation module for $\sigma$.

For each $\lambda \in \mathfrak{a}_{\mathbb{C}}^{*}$, we define

$$
\begin{aligned}
& \mathscr{H}_{\sigma, \lambda}:=\left\{f: G \rightarrow V_{\sigma} \mid f \text { is measurable, } f(\cdot \operatorname{man})=a^{-\rho-\lambda} \sigma(m)^{-1} f(\cdot) \forall \operatorname{man} \in P_{\min },\right. \\
& \left.\qquad \int_{K}\langle f(k), f(k)\rangle_{\sigma} d k<\infty\right\} .
\end{aligned}
$$

The group $G$ acts on $\mathscr{H}_{\sigma, \lambda}$ by left translations, and the Hilbert-space representation thus obtained will be denoted by $\pi_{\sigma, \lambda}$.

Remark 5.1. The relationship between the representations on $\mathscr{H}_{\sigma, \lambda}$ and $L^{2}(\Xi)_{\lambda}$ is as follows. If $\mu_{\sigma}$ is an $M_{H}$-fixed element in $V_{\sigma}^{*}$ (unique up to a scalar factor), then the mapping

$$
\mathscr{H}_{\sigma, \lambda} \rightarrow L^{2}(\Xi)_{\lambda}, \quad f \mapsto \mu_{\sigma}(f)
$$

is a $G$-equivariant injection. This mapping can be made isometric by an appropriate scaling of $\mu_{\sigma}$. By stage induction, one therefore obtains an isometric identification

$$
\widehat{\bigoplus}_{\sigma \in \widehat{M / M_{H}}} \mathscr{H}_{\sigma, \lambda}=L^{2}(\Xi)_{\lambda}
$$

Sometimes, it is useful to realize $\mathscr{H}_{\sigma, \lambda}$ as $V_{\sigma}$-valued functions on $\bar{N}:=\theta(N)$; we then speak of a noncompact realization. Define a weight function on $\bar{N}$ by

$$
w_{\lambda}(\bar{n})=a^{2 \operatorname{Re} \lambda}
$$

where $a \in A$ is determined by the condition $\bar{n} \in K a N$. Then the mapping

$$
\mathscr{H}_{\sigma, \lambda} \rightarrow L^{2}\left(\bar{N}, w_{\lambda}(\bar{n}) d \bar{n}\right) \otimes V_{\sigma},\left.\quad f \mapsto f\right|_{\bar{N}},
$$

is an isometric isomorphism.

Note that

- $\pi_{\sigma, \lambda}$ is irreducible for generic $\lambda$.

- $\pi_{\sigma, \lambda}$ is unitary for $\lambda \in i \mathfrak{a}^{*}$.

- The dual representation of $\pi_{\sigma, \lambda}$ is canonically isomorphic to $\pi_{\sigma^{*},-\lambda}$; the pairing is given by

$$
\langle f, g\rangle:=\int_{\bar{N}}(f(\bar{n}), g(\bar{n}))_{\sigma} d \bar{n},
$$

where $f \in \mathscr{H}_{\sigma, \lambda}, g \in \mathscr{H}_{\sigma^{*},-\lambda}$, and $(\cdot, \cdot)_{\sigma}$ is the natural pairing between $V_{\sigma}$ and $V_{\sigma}^{*}$.

Next, recall the description of $H$-fixed elements in the distribution module $\left(\mathscr{H}_{\sigma, \lambda}^{\infty}\right)^{*}$. First, for each $w \in \mathscr{W}_{H} \backslash \mathscr{W}$ we set

$$
V^{*}(\sigma, w):=\left(V_{\sigma}^{*}\right)^{w^{-1} M_{H} w} .
$$

Note that this space is one-dimensional. Let

$$
V^{*}(\sigma):=\bigoplus_{w \in \mathscr{W}_{H} \backslash \mathscr{W}} V^{*}(\sigma, w) \simeq \mathbb{C}^{\left|\mathscr{W}_{H} \backslash \mathscr{W}\right|}
$$


For $w \in \mathscr{W}_{H} \backslash \mathscr{W}$, we consider the orthogonal projection

$$
V^{*}(\sigma) \rightarrow V^{*}(\sigma, w), \quad \eta \mapsto \eta_{w},
$$

In what follows, we write $\operatorname{Re} \lambda>>0$ if

$$
(\operatorname{Re} \lambda-\rho)\left(\alpha^{\vee}\right)>0 \quad \forall \alpha \in \Sigma^{+} .
$$

Then the formula

$$
j\left(\sigma^{*},-\lambda\right)(\eta)(g)= \begin{cases}a^{-\rho+\lambda} \sigma^{*}\left(m^{-1}\right) \eta_{w} & \text { if } g=h w m a n \in H w M A N \\ 0 & \text { otherwise }\end{cases}
$$

defines a continuous $H$-fixed element in $\mathscr{H}_{\sigma^{*},-\lambda}$ for $\operatorname{Re} \lambda>>0$. We can meromorphically continue $j\left(\sigma^{*}, \cdot\right)$ in the variable $\lambda$ and obtain the identity

$$
j\left(\sigma^{*},-\lambda\right)\left(V^{*}(\sigma)\right)=\left(\left(\mathscr{H}_{\sigma, \lambda}^{\infty}\right)^{*}\right)^{H}
$$

for generic $\lambda$. For large $\lambda$, the inverse mapping $j^{-1}$ is given by

$$
\left(\left(\mathscr{H}_{\sigma, \lambda}^{\infty}\right)^{*}\right)^{H} \ni \nu \mapsto(\nu(w))_{w \in \mathscr{W}_{H} \backslash \mathscr{W}} \in V^{*}(\sigma) .
$$

For a smooth vector $v \in \mathscr{H}_{\sigma, \lambda}$ and $\eta \in V\left(\sigma^{*}\right)$, we obtain a smooth function on $Y=G / H$ by setting

$$
F_{v, \eta}(g H)=\left\langle\pi_{\sigma, \lambda}\left(g^{-1}\right) v, j\left(\sigma^{*},-\lambda\right)(\eta)\right\rangle .
$$

By the Plancherel theorem for $L^{2}(Y)_{\mathrm{mc}}$ (e.g., see [1]), there exists a meromorphic mapping

$$
\mathfrak{a}_{\mathbb{C}}^{*} \rightarrow \operatorname{Gl}\left(V\left(\sigma^{*}\right)\right), \quad \lambda \mapsto C(\sigma, \lambda),
$$

such that the mapping

$$
\Phi: \widehat{\bigoplus}_{\sigma \in \widehat{M / M_{H}}} \int_{i \mathfrak{a}_{+}^{*}}^{\oplus} \mathscr{H}_{\sigma, \lambda} \otimes V^{*}(\sigma) d \lambda \rightarrow L^{2}(Y)_{\mathrm{mc}}
$$

defined on the smooth vectors by the formula

$$
\sum_{\sigma}\left(v_{\sigma, \lambda} \otimes \eta\right)_{\lambda} \mapsto\left(g H \mapsto \sum_{\sigma} \int_{i \mathfrak{a}_{+}^{*}} F_{v_{\sigma, \lambda}, j^{0}\left(\sigma^{*},-\lambda\right)(\eta)}(g H) d \lambda\right),
$$

where $j^{0}(\sigma, \lambda):=j(\sigma, \lambda) \circ C(\sigma, \lambda)$, extends to be a unitary $G$-equivalence. Here $\mathfrak{a}_{+}^{*}$ is a Weyl chamber in $\mathfrak{a}^{*}$.

Remark 5.2. Suppose that $\mathscr{W}=\mathscr{W}_{H}$. (This happens in the group case.) Then $V\left(\sigma^{*}\right)$ is one-dimensional, and we can use Remark 5.1 to obtain the following isomorphism:

$$
\widehat{\bigoplus}_{\sigma \in \widehat{M / M_{H}}} \int_{i \mathfrak{a}_{+}^{*}}^{\oplus} \mathscr{H}_{\sigma, \lambda} \otimes V\left(\sigma^{*}\right) d \lambda \simeq \int_{i \mathfrak{a}_{+}^{*}} L^{2}(\Xi)_{\lambda} d \lambda .
$$

Hence we can view $\Phi$ as a mapping defined on a subspace of $L^{2}(\Xi)$.

The inverse of $\Phi$ is the most continuous Fourier transform $\mathscr{F}$ (or $\mathscr{F}_{m c}$ ). For $f \in L^{2}(Y)_{\mathrm{mc}} \cap$ $L^{1}(Y)$, the Fourier transform is given by

$$
\mathscr{F}(f)(\sigma, \lambda, \eta)(g):=\int_{Y} f(y) j^{0}(\sigma, \lambda)(\eta)\left(y^{-1} g\right) d y,
$$

where $\sigma \in \widehat{M / M_{H}}, \lambda \in i \mathfrak{a}^{*}$, and $\eta \in\left(V^{*}(\sigma)\right)^{*} \simeq V\left(\sigma^{*}\right)$. As a last piece of information, we need to relate the Fourier transform and the horospherical transform.

5.1. The relation between the Fourier transform and the horospherical transform. Now we can describe the relation between $\mathscr{R}$ and $\mathscr{F}$. Let $\mathscr{F}_{A}^{w}$ be $\mathscr{F}_{A}$ considered on $\Xi_{w}=G / M_{H} N^{w}$. Let $f \in C_{c}^{\infty}(Y)$. 
We unwind the definitions:

$$
\begin{aligned}
\mathscr{F}(f)(\sigma, \lambda, \eta)(g) & =\int_{Y} f(g y) j^{0}(\sigma, \lambda)(\eta)\left(y^{-1}\right) d y \\
& =\sum_{w \in \mathscr{W} / \mathscr{W}_{H}} \int_{A N M / w M_{H} w^{-1}} f\left(\text { ganmw } \cdot y_{o}\right) j^{0}(\sigma, \lambda)(\eta)\left(w^{-1} m^{-1} a^{-1} n^{-1}\right) d a d n d m \\
& =\sum_{w \in \mathscr{W} / \mathscr{W}_{H}} \int_{A M / w M_{H} w^{-1}} \mathscr{R}_{w}(f)\left(g m a w M_{H} N^{w}\right) a^{\rho+\lambda} j^{0}(\sigma, \lambda)\left(w^{-1} m^{-1}\right) d a d m \\
& =\sum_{w \in \mathscr{W} / \mathscr{W}_{H}} \int_{M / w M_{H} w^{-1}}\left[\mathscr{F}_{A}^{w} \circ \mathscr{R}_{w}\right](f)\left(w^{-1} \lambda, g m w M_{H} N^{w}\right) j^{0}(\sigma, \lambda)\left(w^{-1} m^{-1}\right) d m \\
& =\sum_{w \in \mathscr{W} / \mathscr{W}_{H}} \int_{M / w M_{H} w^{-1}}\left[\mathscr{F}_{A}^{w} \circ \mathscr{R}_{w}\right](f)\left(w^{-1} \lambda, g m w M_{H} N^{w}\right) \sigma(m) j^{0}(\sigma, \lambda)(\eta)\left(w^{-1}\right) d m .
\end{aligned}
$$

Note that $j^{0}(\sigma, \lambda)(\eta)$ is a distribution, so that a priori the evaluation $j^{0}(\sigma, \lambda)(\eta)\left(w^{-1}\right)$ makes sense only if $\operatorname{Re} \lambda$ is sufficiently small. This problem can be overcome by the meromorphic continuation of $j(\sigma, \lambda)$. This meromorphic continuation can actually be obtained by an iterative procedure starting from small $\operatorname{Re} \lambda$ and providing larger values with the use of a differential operator with polynomial coefficients [4]. This allows us to replace $C_{c}^{\infty}(Y)$ by the Schwartz space $\mathscr{S}(Y)$ of rapidly decaying functions. (See [2, Sec. 12]; this space should not be confused with the Harish-Chandra-Schwartz space $\mathscr{C}(Y)$ considered later in this section.) Thus, we have proved the following assertion.

Lemma 5.3. Let $f \in \mathscr{S}(Y)$. Then

$$
\begin{aligned}
\mathscr{F}(f) & (\sigma, \lambda, \eta)(g) \\
= & \sum_{w \in \mathscr{W} / \mathscr{W}_{H}} \int_{M / w M_{H} w^{-1}}\left[\mathscr{F}_{A}^{w} \circ \mathscr{R}_{w}\right](f)\left(w^{-1} \lambda, g m w M_{H} N^{w}\right) \sigma(m) j^{0}(\sigma, \lambda)(\eta)\left(w^{-1}\right) d m
\end{aligned}
$$

for all $\sigma \in \widehat{M / M_{H}}$ and $\lambda \in i \mathfrak{a}_{+}^{*}$.

Remark 5.4. The special case of $\mathscr{W}=\mathscr{W}_{H}$ is of particular interest. Then the above-mentioned formula is simplified as follows:

$$
\mathscr{F}(f)(\sigma, \lambda, \eta)(g)=\int_{M / M_{H}}\left[\mathscr{F}_{A} \circ \mathscr{R}\right](f)\left(\lambda, g m M_{H} N\right) \sigma(m) j^{0}(\sigma, \lambda)(\eta)(\mathbf{1}) d m .
$$

Theorem 5.5. The restriction of $\mathscr{R}$ to $\mathscr{A}_{\mathrm{mc}} \cap \mathscr{S}(Y)$ is injective.

Proof. Let $f \in \mathscr{A}_{\mathrm{mc}} \cap \mathscr{S}(Y)$. Suppose that $\mathscr{R}(f)=0$. By Remark 3.3, we conclude that $\mathscr{R}_{w}(f)=0$ for all $w$. Hence the last lemma implies that $\mathscr{F}(f)=0$. Since the Fourier transform is injective on $\mathscr{S}(Y)$ (see [2, Cor. 12.7]), we obtain $f=0$.

Remark 5.6. It is very likely that $\mathscr{S}(Y) \cap \mathscr{A}_{\mathrm{mc}}$ is dense in $\mathscr{A}_{\mathrm{mc}}$, but I cannot indicate a reference at the moment. Were this established, the theorem above would imply that the restriction of $\mathscr{R}$ to $\mathscr{A}_{\mathrm{mc}}$ is injective.

\subsection{Concluding remarks.}

5.2.1. The group case. It is instructive to see what the results in this paper mean for a semisimple group $G$ viewed as a symmetric space, i.e., represented as

$$
G \simeq(G \times G) / \Delta(G),
$$

where $\Delta(G)=\{(g, g) \mid g \in G\}$ is the diagonal subgroup. If $P=M A N$ is a minimal parabolic subgroup of $G$ and $\bar{P}=M A \bar{N}$ is its standard opposite (i.e. the image under the corresponding Cartan involution), then the parameter space for the horospheres is given by

$$
\Xi=(G \times G) / \Delta(M A)(N \times \bar{N}) .
$$


In this case, our function space $\mathscr{A}$ is the space of analytic vectors for the left-right regular representation of $G \times G$ on $L^{1}(G)$. Thus, for $f \in \mathscr{A}$ one has

$$
\mathscr{R}(f)((g, h) \Delta(M A)(N \times \bar{N}))=\int_{N \times \bar{N}} f\left(g n \bar{n} h^{-1}\right) d n d \bar{n} .
$$

5.2.2. Further steps. Returning to our more general situation of $Y=G / H$, consider the double fibration

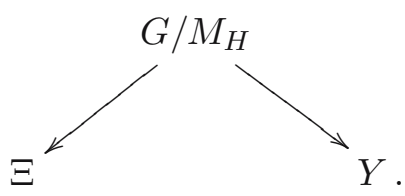

Associated with $\mathscr{R}$ is the dual transform $\mathscr{R}^{\vee}$ between appropriate function spaces $\mathscr{F}(\Xi)$ and $\mathscr{F}(Y)$ on $\Xi$ and $Y$,

$$
\mathscr{F}(\Xi) \rightarrow \mathscr{F}(Y), \quad \mathscr{R}^{\vee}(\phi)(g H)=\int_{H / M_{H}} \phi\left(g h \cdot M_{H} N\right) d\left(h M_{H}\right) .
$$

For $f \in \mathscr{A}_{\mathrm{mc}}$, one can ask whether there exists a pseudo-differential operator $D$ such that $f=$ $\mathscr{R}^{\vee}(D \mathscr{R}(f))$. For $Y=\mathrm{Sl}(2, \mathbb{R}) / \mathrm{SO}(1,1)$, this was considered in [8], where it was shown that $D$ exists. For the case in which $Y$ is a group, one can expect that $D$ is actually a differential operator.

5.2.3. Horospherical transform on Schwartz spaces. One can ask to what extent $\mathscr{R}$ can be defined on the Schwartz space of $Y$. For some classes of spaces $Y$, this seems to be possible, and we comment on this in more detail below. First, recall the definition of the Schwartz space.

We use the decomposition

$$
G=K A H,
$$

often referred to as the polar decomposition of $G$ (with respect to $H$ and $K$ ). Accordingly, every $g \in G$ can be written as $g=k_{g} a_{g} h_{g}$ with $k_{g} \in K$ etc. It is important to note that $a_{g}$ is unique modulo $\mathscr{W}_{H}$. Therefore,

$$
\|g H\|:=\left|\log a_{g}\right| \quad(g \in G)
$$

is well defined provided that $|\cdot|$ is the norm defined by the Killing form on $\mathfrak{p}$. An alternative, often useful description of $\|\cdot\|$ is as follows:

$$
\|y\|=\frac{1}{4}\left|\log \left[y \tau(y)^{-1} \theta\left(y \tau(y)^{-1}\right)^{-1}\right]\right| \quad(y \in Y) .
$$

For $u \in \mathscr{U}(\mathfrak{g})$, let $L_{u}$ be the corresponding differential operator on $Y$; i.e.

$$
\left(L_{u} f\right)(y)=\left.\frac{d}{d t}\right|_{t=0} f(\exp (-t u) y), \quad u \in \mathfrak{g},
$$

for any function $f$ differentiable at $y$. With these preliminaries, one defines the Harish-ChandraSchwartz space of $Y$ as follows:

$$
\mathscr{C}(Y)=\left\{f \in C^{\infty}(Y)\left|\forall u \in \mathscr{U}(\mathfrak{g}) \forall n \in \mathbb{N} \sup _{y \in Y} \Theta(y)(1+\|y\|)^{n}\right|\left(L_{u} f\right)(y) \mid<\infty\right\},
$$

where $\Theta(g H)=\phi_{0}\left(g \tau(g)^{-1}\right)^{-1 / 2}$ and $\phi_{0}$ is the Harish-Chandra basis spherical function.

It is easily seen that $\mathscr{C}(Y)$ equipped with an obvious family of defining seminorms is a Fréchet space. Moreover, it is $G$-invariant, and the action of $G$ on $\mathscr{C}(Y)$ is smooth. Note that $\mathscr{C}(Y) \subset L^{2}(Y)$ is a dense subspace.

Let $B C^{\infty}(\Xi)$ be the space of bounded smooth functions on $\Xi$.

In the context of the definition of $\mathscr{R}$ on $\mathscr{C}(Y)$, consider the following basic example.

Lemma 5.7. Let $Y=\mathrm{Sl}(2, \mathbb{R}) / \mathrm{SO}(1,1)$ and $f \in \mathscr{C}(Y)$. Then the following assertions hold:

(i) The integral $\int_{N} f(n H) d n$ is absolutely convergent.

(ii) The formula

$$
g M_{H} N \mapsto \int_{N} f(g n H) d n
$$


defines a function in $B C^{\infty}(\Xi)$.

Proof. Let $A$ be the diagonal subgroup of $G$ (with positive entries), and let $N=\left(\begin{array}{cc}1 & \mathbb{R} \\ 0 & 1\end{array}\right)$.

(i) For $x \in \mathbb{R}$ and $n_{x}=\left(\begin{array}{ll}1 & x \\ 0 & 1\end{array}\right)$, we should determine $a_{x} \in A$ such that $n_{x} \in K a_{x} H$. We use (5.3) and start:

$$
z_{x}:=n_{x} \tau\left(n_{x}\right)^{-1}=\left(\begin{array}{cc}
1 & x \\
0 & 1
\end{array}\right) \cdot\left(\begin{array}{cc}
1 & 0 \\
-x & 1
\end{array}\right)=\left(\begin{array}{cc}
1-x^{2} & -x \\
x & 1
\end{array}\right)
$$

and hence

$$
y_{x}:=z_{x} \theta\left(z_{x}\right)^{-1}=\left(\begin{array}{cc}
1-x^{2} & -x \\
x & 1
\end{array}\right) \cdot\left(\begin{array}{cc}
1-x^{2} & x \\
-x & 1
\end{array}\right)=\left(\begin{array}{cc}
\left(1-x^{2}\right)^{2}+x^{2} & * \\
* & 1+x^{2}
\end{array}\right) .
$$

For large $|x|$, we have $\log \left|y_{x}\right|=\left|\log y_{x}\right|$. Furthermore, up to an irrelevant constant,

$$
\left|y_{x}\right|=\left[\operatorname{tr}\left(y_{x} y_{x}\right)\right]^{1 / 2} \geqslant \frac{1}{2}\left[\left(1-x^{2}\right)^{2}+x^{2}+1+x^{2}\right] \geqslant \frac{1}{2}\left[x^{4}+1\right] .
$$

Therefore, for large $|x|$ we have

$$
\left\|n_{x}\right\| \geqslant \frac{1}{4} \log \left(x^{4} / 2+1 / 2\right) .
$$

From Harish-Chandra's basic estimates of $\phi_{0}$ and our computation of $z_{x}$, we further obtain $\Theta\left(n_{x}\right) \geqslant|x|$. Therefore, for $f \in \mathscr{C}(Y)$ the function $x \mapsto\left|f\left(n_{x} H\right)\right|$ grows slower than $1 /\left(|x| \cdot|\log x|^{N}\right)$ with any fixed $N>0$ for large $|x|$. This shows (i).

(ii) Let $f \in \mathscr{C}(Y)$ and set $F:=\mathscr{R}(f)$. From the proof of (i), we know that $F$ is smooth. It remains to show that $F$ is bounded. Since $G=K A H$, we see that it suffices to show that $\left.F\right|_{A}$ is bounded. We do so by a straightforward computation. For $t>0$, set

$$
a_{t}=\left(\begin{array}{cc}
t & 0 \\
0 & 1 / t
\end{array}\right) .
$$

Then

$$
a_{t} n_{x}=\left(\begin{array}{cc}
t & t x \\
0 & 1 / t
\end{array}\right)
$$

and hence

$$
z_{t, x}:=a_{t} n_{x} \tau\left(a_{t} n_{x}\right)^{-1}=\left(\begin{array}{cc}
t & t x \\
0 & 1 / t
\end{array}\right) \cdot\left(\begin{array}{cc}
t & 0 \\
-t x & 1 / t
\end{array}\right)=\left(\begin{array}{cc}
t^{2}\left(1-x^{2}\right) & -x \\
x & 1 / t^{2}
\end{array}\right) .
$$

It follows that

$$
y_{t, x}=z_{t, x} \theta\left(z_{t, x}\right)^{-1}=\left(\begin{array}{cc}
t^{4}\left(1-x^{2}\right)^{2}+x^{2} & * \\
* & 1 / t^{4}+x^{2}
\end{array}\right) .
$$

For $t \geqslant 1$, we conclude that

$$
\left\|a_{t} n_{x}\right\| \gtrsim \log \left(\begin{array}{ll}
c_{1} t^{4} & \text { if }|x| \leqslant 1 / 2, \\
c_{2} t^{4} x^{4}-c_{3} & \text { if }|x| \geqslant 1 / 2
\end{array}\right),
$$

and for $|t|<1$ one has

$$
\left\|a_{t} n_{x}\right\| \geqslant \log |x|
$$

From this, we obtain (ii).

This example is somewhat specific. One might expect that the horospherical transform on $\mathscr{C}(Y)$ converges whenever the real ranks of $G$ and of $Y$ coincide.

For groups, it is not hard to show that $\mathscr{R}(f)$ does not converge for general $f \in \mathscr{C}(G)$; the integrability of $f$ is needed. 


\section{References}

[1] E. P. van den Ban and H. Schlichtkrull, "The most continuous part of the Plancherel decomposition for a reductive symmetric space," Ann. of Math., 145:2 (1997), 267-364.

[2] E. P. van den Ban and H. Schlichtkrull, "Fourier inversion on a reductive symmetric space," Acta Math., 182:1 (1999), 25-85.

[3] E. P. van den Ban and H. Schlichtkrull, "The Plancherel decomposition for a reductive symmetric space. II. Representation theory," Invent. Math., 161:3 (2005), 567-628.

[4] I. N. Bernstein, "Analytic continuation of generalized functions with respect to a parameter," Funkts. Anal. Prilozhen., 6:4 (1972), 26-40; English transl.: Functional Anal. Appl., 6:4 (1972), 273-285.

[5] P. Delorme, "Formule de Plancherel pour les espaces symétriques réductifs," Ann. of Math. (2), 147:2 (1998), 417-452.

[6] I. Gelfand and M. Graev, "Geometry of homogeneous spaces, representations of groups in homogeneous spaces and related questions of integral geometry. I," Trudy Moskov. Mat. Obshch., 8 (1959), 321-390.

[7] S. Gindikin, B. Krötz, and G. Ólafsson, "Holomorphic $H$-spherical distribution vectors in principal series representations," Invent. Math., 158:3 (2004), 643-682.

[8] S. Gindikin, B. Krötz, and G. Ólafsson, "Holomorphic horospherical transform on noncompactly causal spaces," Internat Math. Res. Not., 2006, Art. ID 76857.

[9] S. Gindikin, B. Krötz, and G. Ólafsson, "Horospherical model for holomorphic discrete series and horospherical Cauchy transform," Compos. Math., 142:4 (2006), 983-1008.

[10] A. Knapp, Lie Groups beyond an Introduction, 2nd edition, Birkhäuser, Boston, MA, 2002.

[11] B. Krötz and H. Schlichtkrull, On function spaces on symmetric spaces, http://arxiv . org/abs/0711.1087.

[12] T. Matsuki, "The orbits of affine symmetric spaces under the action of minimal parabolic subgroups," J. Math. Soc. Japan, 31:2 (1979), 331-357.

[13] H. Schlichtkrull, Hyperfunctions and Harmonic Analysis on Symmetric Spaces, Progress in Math., vol. 49, Birkhäuser, Boston, MA, 1984.

[14] N. Wallach, Real Reductive Groups. I. Pure and Applied Math., vol. 132, Academic Press, Boston, MA, 1988.

MaX-Planck-Institut Für Mathematik

e-mail: kroetz@mpim-bonn.mpg.de 